\section{Essential elements of biodiversity?}

\section{Introduction to Conservation Genetics}

R Frankham, JD Ballou, DA Briscoe and KH McInness Cambridge University Press, Cambridge, UK. 2002; 617 pp. $£ 34.95$, paperback. ISBN 0-521-63985-9

Heredity (2003) 90, 413-414. doi:10.1038/sj.hdy.6800227

\section{Reviewed by M. Bruford}

This book is the first text book in the relatively young field of conservation genetics and as such is a timely addition to the literature, which until now has comprised a collection of edited volumes and practical manuals, recently augmented by the journal Conservation Genetics itself. The authors have aimed this book at as wide an audience as possible, including undergraduate, Master's and PhD students, conservation biologists and practitioners. As such, it makes a laudable attempt to promulgate a set of ideas that have not been without controversy in the past 10 years. Indeed, the very need for genetic considerations to be factored into conservation planning at a practical level has been, and continues to be, a major debate among those establishing policy and implementing programmes. The book assumes some basic knowledge of Mendelian genetics, but little else is taken for granted and as such it takes an extraordinarily comprehensive and holistic approach to the subject. This approach has resulted in a weighty (and for students, pricey) tome exceeding 500 pages of text, which covers in some detail areas such as molecular markers, quantitative genetics, and population and habitat viability analysis. It is very nicely presented with excellent illustrations and uses boxes, case studies and theory sections judiciously to demonstrate recent advances and both new and classical approaches in conservation genetics.

The book comprises 20 chapters, divided into four sections, starting with an introductory section, which highlights the basic issues concerning conservation biologists today and the potentially important role that genetics can play in the conservation of biological diversity. This section is, in many ways, a justification of the field and its increasing role in conservation and presents recent and convincing arguments for a direct link between genetic diversity and extinction.

The second section (actually called Section I), entitled 'Evolutionary genetics of natural populations', contains much of the basic information that equips the reader for the more applied sections later in the book. It includes comprehensive sections on molecular markers, population genetics, evolution and adaptation, evolutionary forces in small populations and the processes that maintain variation in populations. It is an extremely comprehensive section, which is considerably stronger in theoretical and quantitative genetics than on the molecular biology. This is no bad thing in my view, given the plethora of books already available in molecular evolutionary genetics and the fact that there has been a tendency to view molecular markers as a panacea in conservation biology, which has elevated their use beyond the fact that they are simply one set of tools of many now available in conservation. This section is very dense however and, at nearly 180 pages, not for the faint hearted. While it is certainly true that many conservation students seem to have studiously avoided genetics until the time they come to be exposed to it at final year or Master's level, I do wonder whether some of the sections on evolution in natural populations are too detailed and heavy going in their attempt to be comprehensive.

The third part of the book (Section II), entitled 'Effects of population size reduction', gets to the heart of the matter, including sections on loss of genetic diversity and its implications for fitness, a very comprehensive 50-page section on inbreeding and inbreeding depression, a section on population fragmentation, gene-flow and divergence and finishes with perhaps the nicest section of the book on genetic viability, population size and evolutionary potential. Again, although in parts excessively detailed for all except the most ardent of students, it is nonetheless compelling stuff, about which the authors convey an almost missionary zeal. On page 357 (paperback) in the summary of this section, the authors exhort us to '..not let the occasional persistence of genetically impoverished populations mislead us. We wish to conserve the majority of threatened species, rather than permitting the extinction of many species and conserving only a few exceptional cases.' In keeping with the authors' convictions, there are no half-measures with this book!

In the final section (III) 'From theory to practice' both the practitioner and the student are taken through the practical applications of conservation genetics at the taxonomic level, including discussions on speciation, phylogenetics and defining units for conservation. Unusually for this book, I found the treatment of this subject disappointingly lacking in detail and critique, especially since it is a subject that has been widely and controversially discussed in the conservation literature for the last decade. Further chapters in this section descend the evolutionary hierarchy through population management, captive propagation and reintroduction, and a nice chapter on forensic approaches to conservation that includes examples such as detecting the sale of whale meat from protected species, noninvasive genetic census and the use of the coalescent to infer demographic history of endangered populations. Almost inevitably, given the format the authors have chosen, there is a feeling of déjà $v u$ when reading parts of this section, since there are some strong elements of overlap with Sections I and II, and this is the main disadvantage of the organisational approach they have taken.

The final chapter concerns population viability analysis (PVA), which is a general tool that includes stochastic modelling of demographic, environmental and genetic parameters to assist in conservation planning by attempting to determine which life history, management or other extrinsic factors are likely to have the greatest impact on the survival and evolutionary potential of endangered populations. It is a neat way to finish the book since it demonstrates the way in which predicting the probability of persistence of endangered species is a 
complex exercise, often requiring much uncollected information and because it illustrates the way in which genetics can both inform and be affected by applied management decisions.

So how does this book serve its target audience? Unfortunately, it does not fulfil its brief entirely, in particular I think it is much too detailed for most undergraduate conservation biology courses, although sections of it are invaluable and not found anywhere else. Any Master's level courses in conservation genetics may well adopt the book although perhaps some of it is not quite detailed enough to serve as a core text for graduate school/PhD level. However, Section III should be required reading for conservation practitioners and indeed Master's level conservation biologists. Personally I think it is an excellent achievement and a wide scope, up-to-date piece of scholarship in the truest sense. Finally, it is a testament to the authors that they are able to convey both their reverence for biological diversity, and the sense of urgency needed to devise more rational ways to conserve it at the genetic level, on each and every page.

M Bruford

Cardiff School of Biosciences, Main Building, Museum Avenue, PO Box 915, Cardiff CF10 3TL, UK E-mail: BrufordMW@Cardiff.ac.uk 